\title{
UNIFORM FINITE GENERATION OF LIE GROUPS LOCALLY-ISOMORPHIC TO SL $(2, R)$
}

\author{
RICHARD M. KOCH AND FRANKLIN LOWENTHAL
}

\begin{abstract}
Let $G$ be a connected Lie group with Lie algebra $g,\left\{X_{1}, \cdots, X_{\ell}\right\}$ a minimal generating set for $g$. The order of generation of $G$ with respect to $\left\{X_{1}, \cdots, X_{\ell}\right\}$ is the smallest integer $n$ such that every element of $G$ can be written as a product of $n$ elements taken from $\exp \left(t X_{1}\right), \cdots, \exp \left(t X_{\ell}\right) ; n$ may equal $\infty$. We find all possible orders of generation for all Lie groups locally isomorphic to $S L(2, R)$.
\end{abstract}

1. Introduction. A connected Lie group $G$ is generated by oneparameter subgroups $\exp \left(t X_{1}\right), \cdots, \exp \left(t X_{\ell}\right)$ if every element of $G$ can be written as a finite product of elements chosen from these subgroups. In this case, define the order of generation of $G$ to be the least positive integer $n$ such that every element of $G$ possesses such a representation of length at most $n$; if no such integer exists, let the order of generation of $G$ be infinity. The order of generation will, of course, depend upon the one-parameter subgroups.

Computation of the order of generation of $G$ for given $X_{1}, \cdots, X_{\ell}$ is equivalent to finding the greatest wordlength needed to write each element of a finite group in terms of generators $g_{1}, \cdots, g_{\ell}$. In both cases it is natural to restrict attention to minimal generating sets. From now on, therefore, suppose that no subset of $\left\{\exp \left(t X_{1}\right), \cdots, \exp \left(t X_{\ell}\right)\right\}$ generates $G$.

It is easy to see that $\exp \left(t X_{1}\right), \cdots, \exp \left(t X_{\ell}\right)$ generate $G$ just in case $X_{1}, \cdots, X_{\ell}$ generate the Lie algebra $g$ of $G$. If $\sigma$ is an automorphism of $G$, the order of generation of $G$ with respect to $X_{1}, \cdots, X_{\ell}$ is clearly the same as the order of generation of $G$ with respect to $\sigma_{*}\left(X_{1}\right), \cdots, \sigma_{*}\left(X_{\ell}\right)$. Call two generating sets $\left\{X_{1}, \cdots, X_{\ell}\right\}$ and $\left\{Y_{1}, \cdots, Y_{\ell}\right\}$ equivalent if it is possible to find an automorphism $\sigma$ of $G$, a permutation $\tau$ of $\{1,2, \cdots, \ell\}$, and non-zero constants $\lambda_{1}, \cdots, \lambda_{\ell}$ such that $X_{i}=\lambda_{\ell} \sigma_{*}\left(Y_{\tau(i)}\right)$; the order of generation of $G$ depends only on the equivalence class of the generating set.

In a series of previous papers $[2,3,4,5,6]$, the possible orders of generation for all two and three dimensional linear Lie groups were found. The remaining nonlinear groups are

$$
\left\{\left(\begin{array}{lll}
1 & a & b \\
0 & 1 & c \\
0 & 0 & 1
\end{array}\right) \mid a, b, c \in R\right\} /\left(\left(\begin{array}{lll}
1 & 0 & n \\
0 & 1 & 0 \\
0 & 0 & 1
\end{array}\right) \mid n \in Z\right\}
$$

Received by the editors on December 2, 1975.

AMS (MOS) subject classification (1970). Primary 22E15, 22E60; Secondary 20 F05. 
and $\widetilde{S L}(2, R) / k Z$ for $k=0,3,4,5,6, \cdots$, where $\widetilde{S I}(2, R)$ is the universal covering group of $S L(2, R)$. The first of these groups, however, is easily handled by the methods of [2] (see remark $A$ at the end of that paper). We wish now to finish the calculations for Lie groups of dimension $\leqq 3$ by discussing $\widetilde{S L}(2, R) / k Z$.

2. Results. The group $\widetilde{S L}(2, R) / k Z$ is locally isomorphic to $\widetilde{S L}(2, R) / 2 Z=S L(2, R)$; we always identify its Lie algebra with $s \ell(2, R)$, the set of $2 \times 2$ real matrices of trace zero.

THEOREM. The following is a list of all minimal generating sets for $\widetilde{S L}(2, R) / k Z$ up to equivalence, and the corresponding orders of generation of $\widetilde{S L}(2, R) / k Z$. When a group has order of generation $n$, the last column lists those expressions of length $n$ which give the entire group (for instance, XYX means that every element of the group can be written in the form $\left.\exp \left(t_{1} X\right) \exp \left(t_{2} Y\right) \exp \left(t_{3} X\right)\right)$.

Proof. We can suppose $k \neq 1,2$, for $\operatorname{PS} L(2, R)=\widetilde{S L}(2, R) / Z$ was considered in [3] and $S L(2, R)=\widetilde{S L}(2, R) / 2 Z$ was considered in [6] .

In [2] we classified minimal generating sets for $S L(2, R)$. This classification remains valid for $\widetilde{S L}(2, R) / k Z$ since each automorphism of the Lie algebra $1 \ell(2, R)$ comes from an automorphism of $\widetilde{S L}(2, R) / k Z$. Indeed if $\sigma_{*}$ is an automorphism of $1 \ell(2, R), \sigma_{*}$ induces an automorphism $\sigma$ of $\widetilde{S L}(2, R)$ which takes the center $Z$ of $S L(2, R)$ back to itself; hence $\sigma$ takes $k Z$ to $k Z$ and induces an automorphism of $\widetilde{S L}(2, R) / k Z$.

It is easy to dispose of the first three generating sets on our list. Consider first the elliptic-elliptic case. There is a canonical map $\widetilde{S L}(2, R) / k Z \rightarrow \widetilde{S L}(2, R) / Z=P S L(2, R)$, so the order of generation of $\widetilde{S L}(2, R) / k Z$ must be greater than or equal to the corresponding order of generation of $\operatorname{PSL}(2, R)$; this order is $\infty$ by [3] .

Consider next the elliptic-parabolic and elliptic-hyperbolic cases. Expressions of the form $Y X Y$ do not give all of $P S L(2, R)[3]$, so they cannot give all of $\widetilde{S L}(2, R) / k Z$. It suffices to show that every element of $\widetilde{S L}(2, R)$ can be written in the form $X Y X$. Let $g \in \widetilde{S L}(2, R)$ and call the natural map from $\widetilde{S L}(2, R)$ to $P S L(2, R)$ " $\pi$ ". Then $\pi(g)$ can be written in the form $\exp \left(t_{1} X\right) \exp \left(t_{2} Y\right) \exp \left(t_{3} X\right)$ by [3]. Of course exp is the usual map from $\_l(2, R)$ to $\operatorname{PSL}(2, R)$; if by abuse of notation we let it also denote the map from $1 \ell(2, R)$ to $\widetilde{S L}(2, R)$, then $\pi(g)=$ $\pi\left(\exp \left(t_{1} X\right) \exp \left(t_{2} Y\right) \exp \left(t_{3} X\right)\right)$ and so $g=n \exp \left(t_{1} X\right) \exp \left(t_{2} Y\right) \exp \left(t_{3} X\right)$ where $n \in \operatorname{Ker} \pi$. However, we will show in the next paragraph that every element of $\operatorname{Ker} \pi$ can be written in the form $\exp (t X)$ for some $t$, so $g=\exp (t X) \exp \left(t_{1} X\right) \exp \left(t_{2} Y\right) \exp \left(t_{3} X\right)=\exp \left(\left[t+t_{1}\right] X\right) \exp \left(t_{2} Y\right)$ $\exp \left(t_{3} X\right)$.

If $G$ is an arbitrary connected Lie group with universal covering 
group $\tilde{G}$ and covering map $\pi: \tilde{G} \rightarrow G$, there is a canonical isomorphism $\Psi: \pi_{1}(G) \rightarrow \operatorname{Ker} \pi$; if $\nu:[0,1] \rightarrow G$ represents $\xi \in \pi_{1}(G)$ and $\tilde{\nu}:[0,1]$ $\rightarrow \tilde{G}$ is the lift of $\nu$ to $\tilde{G}, \Psi(\xi)=\tilde{\nu}(1)$. In our case the injection

$$
\left\{\left(\begin{array}{rr}
\cos \theta & -\sin \theta \\
\sin \theta & \cos \theta
\end{array}\right)\right\} / \pm I \rightarrow P S L(2, R)
$$

induces an isomorphism of fundamental groups, so $\nu_{n}(t)=\exp (\pi n t X)$ : $[0,1] \rightarrow P S L(2, R)$ represents $n \in Z=\pi_{1}(P S L(2, R))$; by the same abuse of notation used in the previous paragraph, $\exp (\pi n X)$ equals $n \in Z=\operatorname{Ker} \pi$.

The remaining cases require more thought. Recall that $\operatorname{PSL}(2, R)$ acts on the projective line $P^{1}=R \cup\{\infty\}$ by $x \rightarrow(a x+b) /(c x+d)$. Call an ordered triple $\left(x_{1}, x_{2}, x_{3}\right)$ in $P^{1} \times P^{1} \times P^{1}$ oriented if there is a cyclic permutation $\sigma$ such that $-\infty<x_{\sigma(1)}<x_{\sigma(2)}<x_{\sigma(3)} \leqq \infty$. Whenever $\left(x_{1}, x_{2}, x_{3}\right)$ and $\left(y_{1}, y_{2}, y_{3}\right)$ are oriented triples, $\operatorname{PS} L(2, R)$ contains a unique element mapping $x_{i}$ to $y_{i}$.

Fix a point $A \in P^{1}$. The map $g \rightarrow g(A)$ from $P S L(2, R)$ to $P^{1}$ induces an isomorphism of fundamental groups; indeed it is well known that

$$
\left\{\left(\begin{array}{lr}
\cos \theta & -\sin \theta \\
\sin \theta & \cos \theta
\end{array}\right)\right\} / \pm I \rightarrow P S L(2, R)
$$

induces an isomorphism of fundamental groups, and

$$
\left.\left\{\begin{array}{lr}
\cos \theta & -\sin \theta \\
\sin \theta & \cos \theta
\end{array}\right)\right\} / \pm I \rightarrow P S L(2, R) \rightarrow P^{1}
$$

is a homeomorphism.

Recall the isomorphism $\operatorname{Ker}[\pi: \widetilde{S L}(2, R) \rightarrow P S L(2, R)] \cong \pi_{1}$ $(P S L(2, R))$ discussed earlier. Combining it with the above isomorphism, we find a canonical isomorphism $\operatorname{Ker} \pi \cong \pi_{1}\left(P^{1}\right) \cong Z$. If $\tilde{\nu}$ is a path in $\widetilde{S L}(2, R)$ starting at the identity and ending at $n \in \operatorname{Ker} \pi$, $(\pi \circ \nu)(A)$ goes around $P^{1} n$ times.

The universal covering space $L$ of $P^{1}$ is, of course, homeomorphic to the real line. Without describing the covering map $\tau: L \rightarrow P^{1}$ in detail, let us imagine it so chosen that $\tau^{-1}(\infty)$ consists of all integers and $x \rightarrow x+n$ is a covering map whenever $n$ is an integer.

Fix an oriented triple $(A, B, C) \in P^{1} \times P^{1} \times P^{1}$ and a point $A_{L} \in L$ over $A$. Whenever $\nu:[0,1] \rightarrow \widetilde{S L}(2, R)$ is a path starting at the identity, $\pi \circ \nu(1)$ maps $(A, B, C)$ to a unique oriented triple $(a, b, c)$, and $(\pi \circ \nu(t))(A)$ is a path in $P^{1}$ from $A$ to $a$; this path uniquely lifts to a path in $L$ from $A_{L}$ to a point $a_{L}$ over $a$. Occasionally we write $a_{L}(\nu)$ to indicate the dependence of $a_{L}$ on $\nu$.

Suppose $\mu:[0,1] \rightarrow \widetilde{S L}(2, R)$ is a second path starting at the identity. Then $\pi \circ \nu(1)=\pi \circ \mu(1)$ if and only if $\nu$ and $\mu$ are asso- 
ciated with the same triple $(a, b, c)$. In this case $\nu(1)=n \mu(1)$ where $n \in \operatorname{Ker} \pi$, and $a_{L}(\nu)=a_{L}(\mu)+\hat{n}$; we claim $n=\hat{n}$. In fact, let $\sigma(t)$ be the path in $\widetilde{S L}(2, R)$ obtained by tracing $\nu(t)$ and then tracing $n \mu(t)$ backward; $\sigma$ starts at the identity and ends at $n$. Therefore $(\pi \circ \sigma(t))(A)$ goes around $P^{1} n$ times and its lift to $L$ starts at $A_{L}$ and ends at $A_{L}+n$. But $(\pi \circ \sigma(t))(A)$ is just $(\pi \circ \nu(t))(A)$ followed by $(\pi \circ n \mu(t))(A)$ traced backward. The lift of the first path begins at $A_{L}$ and ends at $a_{L}(\nu)$; the lift of the second path begins at $a_{L}(\mu)$ and ends at $A_{L}$. Equivalently we can lift the second path so that it begins at $a_{L}(\nu)=a_{L}(\mu)+\hat{n}$ and ends at $A_{L}+\hat{n}$, so $\hat{n}=n$.

Consider the expression $\exp \left(t_{1} X_{1}\right) \cdots \exp \left(t_{\ell} X_{\ell}\right)$ in $\widetilde{S L}(2, R)$, where $X_{1}, \cdots, X_{\ell}$ are elements of $1 \ell(2, R)$, not necessarily distinct. There is an obvious path from the identity to this element obtained by setting $t_{1}=\cdots=t_{\ell}=0$ initially, then gradually changing $t_{\ell}$ to its final value, then changing $t_{\ell-1}$ from 0 to its final value, etc. Therefore, $\exp \left(t_{1} X_{1}\right) \cdots \exp \left(t_{\ell} X_{\ell}\right)$ is associated with an oriented triple $(a, b, c)$ and a point $a_{L} \in L$. Indeed, $(A, B, C)$ is mapped to $(a, b, c)$ by moving it first via $X_{\ell}$ to a triple $\left(a_{\ell-1}, b_{\ell-1}, c_{\ell-1}\right)$, then moving $\left(a_{\ell-1}, b_{\ell-1}, c_{\ell-1}\right)$ to $\left(a_{\ell-2}, b_{\ell-2}, c_{\ell-2}\right)$ by $X_{\ell-1}$, and so forth, until finally $\left(a_{1}, b_{1}, c_{1}\right)$ is moved to $(a, b, c)$ by $X_{1}$. Moreover, $A_{L}$ is simultaneously moved to $a_{L}$ by the lifted actions of the $\exp \left(t X_{i}\right)$ on $L$.

If we are given a family of expressions $\left\{\exp \left(t_{1} X_{1}\right) \cdots \exp \left(t_{\ell} X_{\ell}\right), \cdots\right\}$ every element of $\widetilde{S L}(2, R) / k Z$ can be written in one of these forms just in case $(A, B, C)$ can be carried to any oriented triple $(a, b, c)$ by a series of motions " $X_{\ell}$, then $X_{\ell-1}, \cdots$, then $X_{1}$ ", etc., in at least $k$ ways so that the resulting points $a_{L_{1}}, \cdots, a_{L_{k}}$ are inequivalent modulo $k Z$.

After these general remarks, let us turn to a specific example to see how everything works out in practice! Consider the parabolicparabolic case:

$$
X=\left(\begin{array}{ll}
0 & 1 \\
0 & 0
\end{array}\right), \quad Y=\left(\begin{array}{ll}
0 & 0 \\
1 & 0
\end{array}\right)
$$

Since

$$
\exp (t X)=\left(\begin{array}{ll}
1 & t \\
0 & 1
\end{array}\right)
$$

$\exp (t X)(p)=p+t ;$ similarly $\exp (t Y)(p)=p /(p t+1)$. Notice that $\exp (t X)$ leaves $\infty$ fixed and acts transitively on $R ; \exp (t Y)$ leaves 0 fixed and acts transitively on $P^{1}-\{0\}$. Choose the covering map $\tau: L \rightarrow P^{1}$ so that $\tau(1 / 2)=0$. 
LEMMA 1. The order of generation of $\widetilde{S L}(2, R)$ with respect to $X, Y$ is $\infty$; if $k \geqq 2$ is even, the order of generation of $\widetilde{\mathrm{SL}}(2, R) / k Z$ with respect to $X, Y$ is at least $k+2$.

$X:$

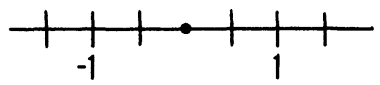

YX:

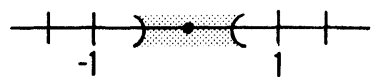

XYX:

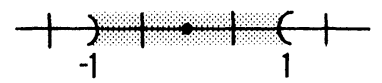

Y:

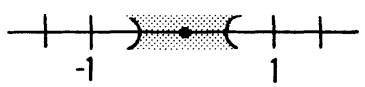

$X Y:$

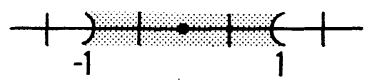

YXY:

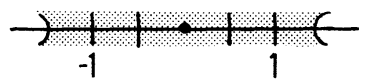

Figure 1

Proof. We will show that it takes at least $k+2$ terms to produce $k$ points in $\pi^{-1}(e)$. Choose $(A, B, C)=(\infty, 0,1), A_{L}=0$; then $(a, b, c)$ also equals $(\infty, 0,1)$. The successive images of $A_{L}$ in $L$ must belong to the shaded regions above. The only way we can get $k$ integral points in the union of the shaded regions associated with expressions with fewer than $k+2$ terms is to use at least one of the integral points at the extremes of the shaded region $(-(k+1) / 2,(k+1) / 2)$ belonging to the expression $Y \cdots Y$ with $k+1$ terms. However, neither of these points can come from an expression mapping $(\infty, 0,1)$ to $(\infty, 0,1)$. For instance, consider the point at the right of the region; let $B_{L}=1 / 2$ in $L$ and watch $B_{L}$ move under the series of maps being considered. Each map preserves order in $L$, so $B_{L}$ must move even further to the right than $k / 2$. But $Y$ leaves $B_{L}$ fixed, $X Y$ moves it into $(0,1) \subset(-1,1), Y X Y$ moves it into $(-3 / 2,3 / 2)$, etc., so the image of $B_{L}$ is in $(-(k+1) / 2,(k+1) / 2)$ and there is no point in $(k / 2,(k+1) / 2)$ equivalent to $1 / 2$.

LEMMA 2. If $k \geqq 3$ is odd, the order of generation of $\widetilde{S L}(2, R) / k Z$ with respect to $X, Y$ is at least $k+2$.

Proof. We will show that it takes at least $k+2$ terms to produce $k$ points in

$$
\pi^{-1}\left(\left(\begin{array}{rr}
0 & -1 \\
1 & 0
\end{array}\right)\right)
$$

Choose $(A, B, C)=(\infty, 0,1), A_{L}=0$; then $(a, b, c)=(0, \infty,-1)$. The only way we can get $k$ half integral points in the union of the shaded regions associated with expressions with fewer than $k+2$ terms is to use at least one of the half integral points at the extremes of the region $(-(k+1) / 2,(k+1) / 2)$ belonging to the expression $X \cdots Y$ with $k+1$ 
terms. Exactly as before, neither of these can come from an expression mapping $(\infty, 0,1)$ to $(0, \infty,-1)$.

Lemma 3. Let $(a, b, c)$ be an oriented triple such that $a \neq \infty$. There is an expression of the form XYX taking $(\infty, 0,1)$ to $(a, b, c)$ and $A_{L}=0$ to $a_{L} \in(-1,0)$, and a second such expression taking $A_{L}$ to $a_{L} \in(0,1)$.

Proof. It is easier to work backward. Note that $X$ applied to $(a, b, c)$ given $(a-\lambda, b-\lambda, c-\lambda)$. If eventually $(a, b, c)$ is to go to $(\infty, 0,1)$, $Y$ must take $a-\lambda$ to $\infty$ since $\infty$ is a fixed point of $X$. Therefore

$$
Y(p)=\frac{p}{1-\frac{p}{a-\lambda}}
$$

and $Y X$ maps $(a, b, c)$ to $(\infty,(a-\lambda)(b-\lambda) /(a-b),(a-\lambda)(c-\lambda) /(a$ $-c)$ ). A final translation can carry this to $(\infty, 0,1)$ just in case

$$
\left|\frac{(a-\lambda)(b-\lambda)}{a-b}-\frac{(a-\lambda)(c-\lambda)}{a-c}\right|=1
$$

(remember that all triples are oriented). So we want to choose $\lambda$ such that $|a-\lambda|^{2}|(b-c) /(a-b)(a-c)|=1$; this is possible in exactly two ways. For one of the two ways $a-\lambda<0$, so $a_{L} \in(0,1)$; for the other $a-\lambda>0$ and $a_{L} \in(-1,0)$.

LEMMA 4. If $k \geqq 2$, every element of $\widetilde{S L}(2, R) / k Z$ can be written in the form $\cdots X Y X$ using $k+2$ terms.

Proof. As usual let $(A, B, C)=(\infty, 0,1), A_{L}=0$. Let $(a, b, c)$ be an arbitrary oriented triple. The earlier picture shows that we can map $A_{L}$ to $k$ elements $a_{L}$ in $L$ covering $a$, inequivalent modulo $k Z$, by expressions $\cdots X Y X$ with at most $k+2$ terms. Consider a typical such expression and assume that no term is the identity. Its inverse carries $(a, b, c)$ to $(\infty, \beta, \nu)$ and its last three terms $X Y X$ carry $(\infty, \beta, \nu)$ to $\left(\alpha_{1}, \beta_{1}, \nu_{1}\right)$. The element $a_{L, 1}$ in $L$ over $\alpha_{1}$ belongs to $(-1,1)$. Note that $\alpha_{1} \neq \infty$, for otherwise $X Y X$ carries $A_{L}$ back to itself and this would require $Y$ to be the identity. Now by lemma 3 there is a second expression $\widetilde{X Y X}$ carrying $(\infty, 0,1)$ to $\left(\alpha_{1}, \beta_{1}, \nu_{1}\right)$ and $A_{L}$ to $a_{L, 1}$; replacing $\cdots Y(X Y X)$ by $\cdots Y(\widetilde{X Y X})$, we obtain an expression that maps $(\infty, 0,1)$ to $(a, b, c)$ and $A_{L}$ to $a_{L}$.

LEMMA 5. If $k \geqq 2$, every element in $\widetilde{S L}(2, R) / k Z$ can be written in the form $\cdots$ YXY with $k+2$ terms.

Proof. There is an automorphism $\sigma$ of $\iota \ell(2, R)$ interchanging $X$ and $Y$ up to scalars; indeed $\sigma(A)=-A^{T}$. Thus lemma 4 implies lemma 5. 
REMArK. Next consider the parabolic-hyperbolic case:

$$
X=\left(\begin{array}{ll}
0 & 1 \\
0 & 0
\end{array}\right), Y=\left(\begin{array}{ll}
0 & 1 \\
1 & 0
\end{array}\right) .
$$

Notice that $\exp (t Y)$ leaves two points \pm 1 in $P^{1}$ fixed and acts transitively on each of the connected components of $P^{1}-\{ \pm 1\}$. We can suppose $\tau(1 / 3)=-1, \tau(2 / 3)=1$.

LEMMA 6. The order of generation of $\widetilde{S L}(2, R)$ with respect to $X, Y$ is $\infty$; if $k \geqq 2$ is even, the order of generation of $\widetilde{S L}(2, R) / k Z$ with respect to $X, Y$ is at least $k+2$.

Proof. As before, we will show that it takes at least $k+2$ terms to produce $k$ points in $\pi^{-1}(e)$. Choose $(A, B, C)=(\infty,-1,1), A_{L}=0$. The successive images of $A_{L}$ in $L$ must belong to the shaded regions below.

X:

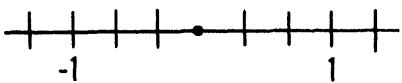

YX:

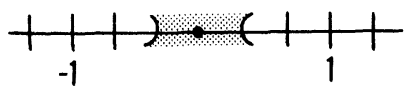

XYX:

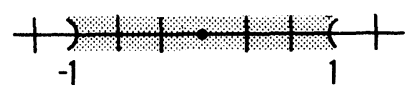

Y:

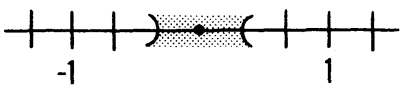

$\mathrm{XY}:$

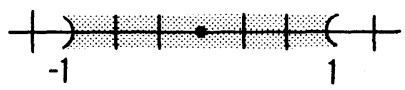

YXY:

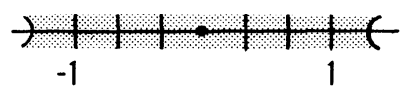

Figure 2

The rest of the argument is exactly as in the proof of lemma 1.

LEMMA 7. If $k \geqq 3$ is odd, the order of generation of $\widetilde{S L}(2, R) / k Z$ with respect to $X, Y$ is at least $k+2$. Indeed, even the expression $Y \cdots Y$ of length $k+2$ cannot give all of $\widetilde{S L}(2, R) / k Z$.

Proof. It suffices to prove the last statement, for any expression of length $k+1$ can be made to look like the expressions $Y \cdots Y$ of length $k+2$ by adding $Y$ at the beginning or the end. Let $(A, B, C)=$ $(-1,1, \infty), \quad A_{L}=1 / 3$, and let $g \in P S L(2, R)$ map this triple to $(1,-1,0)$. Then $Y(1 / 3)=1 / 3, Y(2 / 3)=2 / 3, Y X Y(1 / 3)$ and $Y X Y(2 / 3)$ are contained in $(-1 / 3,4 / 3), Y X Y X Y(1 / 3)$ and $Y X Y X Y(2 / 3)$ are contained in $(-4 / 3,7 / 3)$, etc., so $Y \cdots Y(1 / 3)$ and $Y \cdots Y(2 / 3)$ belong to a region $D$ which contains exactly $k$ points congruent to $2 / 3$. However, the largest of these points cannot come from a map taking $(-1,1, \infty)$ to $(1,-1,0)$ because $Y \cdots Y(2 / 3)$ would be larger than $Y \cdots Y(1 / 3)$ and congruent to $1 / 3$, and there is no such point in $D$. 
REMARK. To finish this case, it is enough to prove that whenever $k \geqq 2$, the expression $\cdots Y Y X Y X$ of length $k+2$ generates $\widetilde{S L}(2, R) / k Z$. Indeed if $k$ is even and $g \in \widetilde{S L}(2, R) / k Z$, write $g^{-1}=Y\left(t_{1}\right) X\left(t_{2}\right) \cdots$ $Y\left(t_{k+1}\right) X\left(t_{k+2}\right)$; then $g=X\left(-t_{k+2}\right) Y\left(-t_{k+1}\right) \cdots X\left(-t_{2}\right) Y\left(-t_{1}\right)$, so $\cdots X Y X Y$ also generates $\widetilde{S L}(2, R) / k Z$.

LemMa 8. Let $(a, b, c)$ be an oriented triple with $a \neq \infty$ and let $a_{L} \in(-1,1)$ cover $a$. There is an expression of the form YXYX mapping $(\infty,-1,1)$ to $(a, b, c)$ and $A_{L}=0$ to $a_{L}$.

Proof. Notice that in $L, Y X Y X(0) \in(-4 / 3,4 / 3)$. Since every element of $S L(2, R)$ can be written in the form YXYX [6], for each oriented triple $(a, b, c)$ there exist two expressions of the form YXYX mapping $(\infty,-1,1)$ to $(a, b, c)$ and taking $A_{L}=0$ to $a_{1}$ and $a_{2}$ respectively, such that $a_{1}$ and $a_{2}$ are inequivalent modulo $2 Z$. But points in $[-2 / 3,2 / 3]$ are equivalent modulo $2 Z$ only to themselves in $(-4 / 3$, $4 / 3$ ), so each element of $[-2 / 3,2 / 3]$ occurs in this way. We must investigate the intervals $(-1,-2 / 3)$ and $(2 / 3,1)$; by symmetry it suffices to study $(2 / 3,1)$.

We shall work backward from $(a, b, c)$ to $(\infty,-1,1)$; since elements in $\exp (t X)$ are translations preserving $\infty$, it suffices to find an expression of the form YXY mapping $(a, b, c)$ to $(\infty, \tilde{c}, \tilde{c})$ and $a_{L}$ to 0 such that $|\tilde{c}-\tilde{b}|=2$. We are already supposing $a_{L} \in(2 / 3,1)$; it is easy to see that after application of a suitable expression in $\exp (t Y)$, we can also suppose that $b$ and $c$ are represented by $b_{L}$ and $c_{L}$ in $L$ such that $0<b_{L}<c_{L}<a_{L}$. Applying a suitable $X$, we can assume $0<b_{L}<$ $c_{L}<a_{L}<1 / 3$. Having now used the first $Y$ and $X$ available to us, we must show that whenever $(a, b, c)$ is an oriented triple covered by $a_{L}, b_{L}, c_{L}$ in $L$ and $0<b_{L}<c_{L}<a_{L}<1 / 3$, there is an expression of the form YX mapping $a_{L}$ to 0 and $(a, b, c)$ to $(\infty, \tilde{b}, \tilde{c})$ such that $|\tilde{b}-\tilde{c}|=2$.

For each $x \in(0,1 / 3)$, there is a unique $t_{1}$ such that $\exp \left(t_{1} X\right)$ maps $a_{L}$ to $x$; let this element map $b_{L}$ and $c_{L}$ to $b_{L}(x)$ and $c_{L}(x)$ covering $b(x)$ and $c(x)$ in $P^{1}$. Notice that $0<b_{L}(x)<c_{L}(x)<x<1 / 3$, so $-\infty<b(x)<c(x)<\tau(x)<-1$. For each $x \in(0,1 / 3)$, there is a unique $t_{2}$ such that $\exp \left(t_{2} Y\right)$ maps $x$ to 0 ; let this element map $b_{L}(x)$ and $c_{L}(x)$ to $\tilde{b}_{L}(x)$ and $\tilde{c}_{L}(x)$ covering $\tilde{b}(x)$ and $\tilde{c}(x)$ in $P^{1}$. Notice that $-1 / 3<b_{L}(x)<\tilde{c}_{L}(x)<0$, so $1<\tilde{b}(x)<\tilde{c}(x)<\infty$. Clearly $\mid \tilde{c}(x)$ $-b(x) \mid$ is a continuous function of $x$. To complete the proof, it is enough to show that $|\tilde{c}(x)-b(x)| \rightarrow 0$ as $x \rightarrow 1 / 3$ and $|\tilde{c}(x)-\tilde{b}(x)| \rightarrow \infty$ as $x \rightarrow 0$.

Whenever $a, b, c$ and $d$ are four distinct points in $P^{1}$, the cross ratio $\langle a, b ; c, d\rangle$ is by definition $(a-c) / a-d) \cdot(b-d) /(b-c)$; recall that 
the action of $P S L(2, R)$ on $P^{1}$ preserves cross ratios. Hence $\langle 1,-1$; $\tau(x), c(x)\rangle=\langle 1,-1 ; \infty, \tilde{c}(x)\rangle$ and $(1-\tau(x) /(1-c(x)) \cdot(-1-c(x) /$ $(-1-\tau(x))=(-1-\tilde{c}(x)) /(1-\tilde{c}(x))$. As $x \rightarrow 1 / 3, \tau(x) \rightarrow-1$; moreover $c(x)-\tau(x)=c-a$ because $\exp (t X)$ is a translation so $c(x)$ $\rightarrow c-a-1 \neq-1$. Hence $(-1-\tilde{c}(x)) /(1-\tilde{c}(x)) \rightarrow \infty$, so $\tilde{c}(x) \rightarrow 1$. Since $1<\tilde{b}(x)<\tilde{c}(x)<\infty,|\tilde{c}(x)-\tilde{b}(x)| \rightarrow 0$.

Next we study the situation as $x \rightarrow 0$. Then $\langle 1,-1 ; \tau(x), c(x)\rangle=$ $\langle 1,-1 ; \infty, \tilde{c}(x)\rangle$ so $(1-\tau(x)) /(1-c(x)) \cdot(-1-c(x)) /(-1-\tau(x))=$ $(-1-\tilde{c}(x)) /(1-\tilde{c}(x))$. If $x \rightarrow 0, c_{L}(x) \rightarrow 0$ since $0<c_{L}(x)<x$, so $\tau(x)$ and $c(x)$ approach $\infty,(1-\tau(x)) /(-1-\tau(x)) \cdot(-1-c(x)) /(1-$ $c(x))=(-1-\tilde{c}(x)) /(1-\tilde{c}(x))$ approaches 1 , and thus $\tilde{c}(x)$ approaches $\infty$. Then $\langle 1, b(x) ; \tau(x), c(x)\rangle=\langle 1, b(x) ; \infty, \tilde{c}(x)\rangle$, so $(1-\tau(x)) /(1-$ $c(x)) \cdot(b(x)-c(x)) /(b(x)-\tau(x))=(b(x)-\tilde{c}(x)) /(1-\tilde{c}(x))$. But each element of $\exp (t X)$ acts on $P^{1}$ by translation, so $(b(x)-c(x)) /(b(x)-$ $\tau(x))$ is a non-zero constant independent of $x$. Similarly $\tau(x)=\tau\left(a_{L}\right)$ $+\lambda(x)$ and $c(x)=\tau\left(c_{L}\right)+\lambda(x)$ where $\lambda(x) \rightarrow \infty$ as $x \rightarrow 0$, so $(1-\tau(x)) /$ $(1-c(x)) \rightarrow 1$ as $x \rightarrow 0$. Consequently $(\tilde{b}(x)-\tilde{c}(x)) /(1-\tilde{c}(x))$ approaches a non-zero constant as $x \rightarrow 0$; since $\tilde{c}(x) \rightarrow \infty,|\tilde{c}(x)-\tilde{b}(x)|$ $\rightarrow \infty$.

LEMma 9. Let $k \geqq 3$. There is an expression $\cdots Y X$ with $k+2$ terms mapping $(\infty,-1,1)$ to $(a, b, c)$ and $A_{L}=0$ to $a_{L}$ provided $a_{L} \in[-k / 2, k / 2]$ if $k$ is even, $a_{L} \in(-(k+1) / 2,(k+1) / 2)$ if $k$ is odd. In particular $\cdots Y$ Y generates $\widetilde{S L}(2, R) / k Z$.

Proof. We prove this by induction on $k$. Lemma 8 suffices to begin the induction because our proof of the step $k \rightarrow k+1$ for $k$ even will only require the induction hypothesis when $a_{L} \in(-k / 2, k / 2)$.

Suppose the theorem is known for an even $k$; we prove it for $k+1$. Let $(a, b, c)$ and $a_{L} \in(-(k / 2)-1,(k / 2)+1)$ be given. It is possible to map $a_{L}$ into the region $(-k / 2, k / 2)$ by an expression of the form $Y_{1}{ }^{-1} X_{1}{ }^{-1}$; suppose that $a_{L}$ goes to $\tilde{a}_{L}$ and $(a, b, c)$ goes to $(\tilde{a}, \tilde{b}, \tilde{c})$. When $k=2$, we can assume $\tilde{a} \neq \infty$. By induction there is an expression $Y X \cdots Y X$ of length $k+2$ taking $(\infty,-1,1)$ to $(\tilde{a}, \tilde{b}, \tilde{c})$ and $A_{L}$ to $\tilde{a}_{L}$. Hence $\left(X_{1} Y_{1}\right)(Y X \cdots Y X)=X_{1}\left(Y_{1} Y\right) X \cdots Y X$ carries $(\infty,-1,1)$ to $(a, b, c)$ and $A_{L}$ to $a_{L}$.

If the theorem is known for an odd $k,(a, b, c)$ is a given triple, and $a_{L} \in[-(k+1) / 2,(k+1) / 2]$, we can find $Y_{1}$ carrying $a_{L}$ to $\tilde{a}_{L}$ in $(-(k+1) / 2,(k+1) / 2)$ and $(a, b, c)$ to $(\tilde{a}, \tilde{b}, \tilde{c})$; by induction there is an expression $X \cdots Y X$ taking $(\infty,-1,1)$ to $(\tilde{a}, \tilde{b}, \tilde{c})$ and $A_{L}$ to $\tilde{a}_{L}$, so $Y_{1} X \cdots Y X$ carries $(\infty,-1,1)$ to $(a, b, c)$ and $A_{L}$ to $a_{L}$.

Remark. Consider next the hyperbolic-hyperbolic (fixed points interlacing) case: 


$$
X=\left(\begin{array}{rr}
1 & 0 \\
0 & -1
\end{array}\right), \quad Y=\left(\begin{array}{rr}
\alpha & 1 \\
1 & -\alpha
\end{array}\right), \alpha \geqq 0 .
$$

Notice that $\exp (t X)$ leaves two points $0, \infty$ fixed and $\exp (t Y)$ leaves two points $\alpha \pm\left(\alpha^{2}+1\right)^{1 / 2}$ fixed; both $\exp (t X)$ and $\exp (t Y)$ act transitively on the connected components of the complements of their fixed point sets. We can suppose $\tau(1 / 4)=\alpha-\left(\alpha^{2}+1\right)^{1 / 2}, \tau(1 / 2)=0, \tau(3 / 4)=$ $\alpha+\left(\alpha^{2}+1\right)^{1 / 2}$. Although we will refrain from drawing orbit pictures from now on, the reader will often find it useful to do so.

Since $\exp (t X)(p)=\mathrm{e}^{2 t} p, \exp (t X)(p)$ approaches 0 as $\tau \rightarrow-\infty$ and $\infty$ as $t \rightarrow \infty$; a similar statement holds for $Y$.

LEMMA 10. The order of generation of $\widetilde{S L}(2, R)$ with respect to $X, Y$ is $\infty$. If $k \geqq 2$ is even, the order of generation of $\widetilde{S L}(2, R) / k Z$ with respect to $X, Y$ is at least $2 k+4$.

Proof. We will show that it takes at least $2 k+4$ terms to produce $k$ points in $\pi^{-1}(e)$. Notice that any expression giving $e$ in $\operatorname{PS} L(2, R)$ must act on $L$ by $x \rightarrow x+n, n \in Z$, since the lift to $L$ of a motion of $P^{1}$ is uniquely determined up to covering transformations and the identity on $L$ is one lift of the identity map on $P^{1}$. Any non-trivial motion of $L$ induced by $\exp (t X)$ or $\exp (t Y)$ maps one of $0,1 / 4,1 / 2,3 / 4$ left and one right; for instance $\exp (t X)$ for $t>0$ acts as follows:

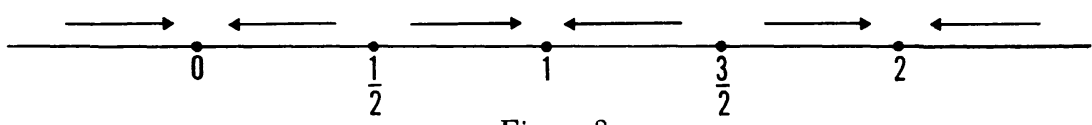

Figure 3

Suppose we are given an expression with fewer than $2 k+4$ terms. Without loss of generality we can suppose that 0 is initially left fixed and then moved left. Thus the expression begins with $X$, and $X(0)=0, Y X(0)<0, X Y X(0)<0, Y X Y X(0)<1 / 4, X Y X Y X(0)<1 / 2$, etc., so that eventually the image of 0 is smaller than $k / 2$. Hence the only translations of $L, x \rightarrow x+n$, that can be achieved are those with $n<k / 2$. Similarly $n$ must be larger than $-k / 2$; there are only $k-1$ integers in the interval $(-k / 2, k / 2)$.

LEMMA 11. If $k \geqq 1$ is odd, the order of generation of $\widetilde{S L}(2, R) / k Z$ with respect to $X, Y$ is at least $2 k+4$.

Proof. We will show that it takes at least $2 k+4$ terms to produce $k$ points in

$$
\pi^{-1}\left(\left(\begin{array}{rr}
0 & -1 \\
1 & 0
\end{array}\right)\right)
$$


Notice that the map $x \rightarrow-1 / x$ interchanges 0 and $\infty$ and also interchanges $\alpha-\left(\alpha^{2}+1\right)^{1 / 2}$ and $\alpha+\left(\alpha^{2}+1\right)^{1 / 2}$. A little thought shows that we can choose the covering map $\tau: L \rightarrow P^{1}$ so that $\tau(x+1 / 2)$ $=-1 / \tau(x)$; thus the lift of

$$
\left(\left(\begin{array}{rr}
0 & -1 \\
1 & 0
\end{array}\right)\right)
$$

to $L$ has the form $x \rightarrow x+1 / 2+n$ for some integer $n$.

The proof of lemma 11 is exactly like the proof of lemma 10 . Given an expression with fewer than $2 k+4$ terms, we can choose one of $0,1 / 4,1 / 2,3 / 4$, say 0 , so that the initial term of the expression leaves 0 fixed and the next moves it to the left; then the final image of 0 is smaller than $k / 2$. Similarly, the final image of 0 is larger than $-k / 2$. The map on $L$ thus has the form $x \rightarrow x+1 / 2+n$ where $|1 / 2+n|<$ $k / 2$, and there are only $k-1$ such integers $n$.

LEMMA 12. If $b$ in $P^{1}$ is not $\infty$, there is an expression of the form YXY taking $\infty$ to $\infty$ and 0 to $b$.

Proof. There is an action of $Y$ on $L$ taking $1 / 2$ to $3 / 8$; denote the image of 0 by $\delta$ and note that $0<\delta<1 / 4$. For each $t \geqq 0,(\exp t X)(\delta)$ belongs to the interval $(0, \delta] \subseteq(0,1 / 4)$ and there is a unique $u(t)$ such that $(\exp u(t) Y \exp t X)(\delta)=0 . \quad$ Let $(\exp u(t) Y \exp t X)(3 / 8)=b_{L}(t)$; $b_{L}(t)$ is continuous in $t$ and $b_{L}(0)=1 / 2$. Notice that as $t \rightarrow \infty, b_{L}(t)$ $\rightarrow 0$. Consequently there is an expression $Y X Y$ taking 0 to 0 and $1 / 2$ to any $b_{L} \in(0,1 / 2]$.

Similarly there is an expression of the form $Y X Y$ taking 0 to 0 and $-1 / 2$ to any $b_{L} \in[-1 / 2,0)$. The lemma follows immediately by projection of these results from $L$ to $P^{1}$.

REMark. The orbit picture shows that the expression $Y X Y$ whose existence is guaranteed by this lemma preserves $\tau^{-1}(\infty)$ in $L$ pointwise.

LEMMA 13. Every element of $\widetilde{S L}(2, R) / k Z$ can be written in terms of the expression $Y X \cdots X$ with $2 k+4$ terms.

Proof. Choose $(A, B, C)=\left(\infty, 0, \alpha+\left(\alpha^{2}+1\right)^{1 / 2},\right), A_{L}=0$. Let $(a, b, c)$ be an oriented triple, $a_{L}$ an element in [ $\left.-k / 2, k / 2\right]$ covering $a$. The orbit picture shows that there is an expression $Y X \cdots X Y$ with $2 k+1$ terms mapping $A_{L}$ to $a_{L}$. Let the inverse of this expression map $(a, b, c)$ to $(\infty, \tilde{b}, \tilde{c})$. By the previous lemma, there is an expression $\widetilde{Y X Y}$ mapping $(\infty, 0)$ to $(\infty, \tilde{b})$. Hence the expression $(Y X \cdots X Y)$ $(\widetilde{Y X Y})=(Y X \cdots X)(Y \tilde{Y})(\widetilde{X Y})$ with $2 k+3$ terms maps $A_{L}$ to $a_{L}$ and $(\infty, 0, \hat{c})$ to $(a, b, c)$ for some $\hat{c} \in(0, \infty)$. There is an $\tilde{\tilde{X}}$ taking $\alpha+$ $\left(\alpha^{2}+1\right)^{1 / 2}$ to $\hat{c}$; then $(Y X \cdots X)(Y \tilde{Y})(\widetilde{X Y}) \tilde{\tilde{X}}$ takes $A_{L}$ to $a_{L}$ and 
$\left(\infty, 0, \alpha+\left(\alpha^{2}+1\right)^{1 / 2}\right)$ to $(a, b, c)$.

LEMMA 14. Every element of $\widetilde{S L}(2, R) / k Z$ can be written in terms of the expression $X Y \cdots Y$ with $2 k+4$ terms.

Proof. If $g \in \widetilde{S L}(2, R) / k Z$, write $g^{-1}=Y\left(t_{1}\right) X\left(t_{2}\right) \cdots X\left(t_{2 k+4}\right)$; then $g=X\left(-t_{2 k+4}\right) \cdots X\left(-t_{2}\right) Y\left(-t_{1}\right)$.

REMARK. Next we consider the hyperbolic-hyperbolic (fixed points noninterlacing) case:

$$
X=\left(\begin{array}{rr}
1 & 0 \\
0 & -1
\end{array}\right), \quad Y=\left(\begin{array}{ll}
\alpha & -1 \\
1 & -\alpha
\end{array}\right), \alpha>1 .
$$

The fixed points of $\exp (t X)$ are $0, \infty$ and those of $\exp (t Y)$ are $\alpha \pm$ $\left(\alpha^{2}-1\right)^{1 / 2}$. We suppose $\tau(1 / 4)=0, \tau(1 / 2)=\alpha-\left(\alpha^{2}-1\right)^{1 / 2}, \tau(3 / 4)$ $=\alpha+\left(\alpha^{2}-1\right)^{1 / 2}$.

LEMMA 15. The order of generation of $\widetilde{S L}(2, R)$ with respect to $X, Y$ is $\infty$. If $k \geqq 2$ is even, the order of generation of $\widetilde{S L}(2, R) / k Z$ with respect to $X, Y$ is at least $k+2$; indeed neither expression of length $k+2$ can give all of $\widehat{S L}(2, R) / k Z$.

Proof. Let $g$ in $\operatorname{PS} L(2, R)$ map $(0,1, \infty)$ to $\left(\alpha-\left(\alpha^{2}-1\right)^{1 / 2}, 1\right.$, $\left.\alpha+\left(\alpha^{2}-1\right)^{1 / 2}\right)$. We will show that the expression $Y X \cdots X$ with length $k+2$ cannot produce $k$ points in $\pi^{-1}(g)$.

Notice that in $L, X(0)=0, Y X(0) \in(-1 / 4,1 / 2), X Y X(0) \in(-3 / 4,1)$, $Y X Y X(0) \in(-5 / 4,3 / 2)$, etc., so the image of 0 under the expression with $k+2$ terms is contained in $(-k / 2-1 / 4, k / 2+1 / 2)$, a region with exactly $k$ points equivalent to $3 / 4$. However, the largest of these points cannot correspond to an expression giving $g$ in $\operatorname{PS} L(2, R)$, since the image of $1 / 4$ would also belong to the region described above, would be larger than the image of 0 , and would be equivalent to $1 / 2$, and there is no such point.

Similarly we can find an element $\tilde{g} \in P S L(2, R)$ such that the expression $X Y \cdots Y$ of length $k+2$ cannot produce $k$ points in $\pi^{-1}(\tilde{g})$.

LEMMA 16. If $k \geqq 3$ is odd, the order of generation of $\widetilde{S L}(2, R) / k Z$ with respect to $X, Y$ is at least $k+2$; indeed neither expression of length $k+2$ can give all of $\widetilde{S L}(2, R) / k Z$.

Proof. Let $g$ in $\operatorname{PS} L(2, R)$ map $(\infty, 0,1)$ to $(0, \infty,-1)$; we will show that the expression $X Y \cdots X$ of length $k+2$ cannot produce $k$ points in $\pi^{-1}(g)$.

Notice that in $L$, the image of 0 under the expression in question is contained in $(-(k+1) / 2+1 / 4,(k+1) / 2)$; this region contains $k$ points equivalent to $1 / 4$. However, the largest of these points cannot 
correspond to an expression giving $g$ in $\operatorname{PS} L(2, R)$, since the image of $1 / 4$ would also belong to the region described above, would be larger than the image of 0 , and would be equivalent to 0 , and there is no such point.

Similarly we can find an element. $\tilde{g} \in P S L(2, R)$ such that the expression $Y X \cdots Y$ of length $k+2$ cannot produce $k$ points in $\pi^{-1}(\tilde{g})$.

REMARK. We now wish to show that the order of generation of $\widetilde{S L}(2, R) / k Z$ with respect to $X, Y$ is exactly $k+2$. As usual, we may assume $k \geqq 3$. Whenever $(a, b, c)$ is an oriented triple of points in $P^{1}$, there is a unique fourth point $d$ such that the element in $\operatorname{PS} L(2, R)$ which maps $\left(\infty, 0, \alpha-\left(\alpha^{2}-1\right)^{1 / 2}\right)$ to $(a, b, c)$ maps $\alpha+\left(\alpha^{2}-1\right)^{1 / 2}$ to $d$. Notice that $g$ in $\operatorname{PSL}(2, R)$ maps $\left(\infty, 0, \alpha-\left(\alpha^{2}-1\right)^{1 / 2}\right)$ to $(a, b, c)$ just in case it maps one of the oriented triples obtained from $(\infty, 0, \alpha-$ $\left.\left(\alpha^{2}-1\right)^{1 / 2}, \alpha+\left(\alpha^{2}-1\right)^{1 / 2}\right)$ by omitting a point to the corresponding triple in $(a, b, c, d)$. The following lemmas show that whenever $(a, b, c)$ is an oriented triple, there is an oriented triple formed by omitting one of the points of $(a, b, c, d)$, say for purposes of discussion $(b, c, d)$, and an expression of length $k+2$ taking $(b, c, d)$ to $\left(0, \alpha-\left(\alpha^{2}-1\right)^{1 / 2}\right.$, $\left.\alpha+\left(\alpha^{2}-1\right)^{1 / 2}\right)$ in $k$ ways so that the element $b_{L}$ in $L$ covering $b$ maps to $k$ elements $1 / 4+n_{1}, \cdots, 1 / 4+n_{k}$ covering 0 and if $i \neq j, n_{i}-n_{j}$ $\notin k Z$. This suffices to prove that $\widetilde{S L}(2, R) / k Z$ has order of generation $k+2$ for the inverses of the expressions in question map $(0, \alpha-$ $\left.\left.\alpha^{2}-1\right)^{1 / 2}, \alpha+\left(\alpha^{2}-1\right)^{1 / 2}\right)$ to $(b, c, d)$ and $1 / 4+n_{i}$ to $b_{L}$ or (by lifting in a different way) $1 / 4$ to $b_{L}-n_{i}$; our previous remarks show that the resulting $k$ elements of $\widetilde{S L}(2, R) / k Z$ are unequal and their projections to $\operatorname{PSL}(2, R) \operatorname{map}\left(\infty, 0, \alpha-\left(\alpha^{2}-1\right)^{1 / 2}\right)$ to $(a, b, c)$.

Notice that the interval $(-\infty, 0)$ in $P^{1}$ is an orbit of $\exp (t X)$ which excludes the fixed points of $Y$; the interval $\left(\alpha-\left(\alpha^{2}-1\right)^{1 / 2}, \alpha+\right.$ $\left.\left(\alpha^{2}-1\right)^{1 / 2}\right)$ plays the same role for $Y$.

LEMMA 17. Let $n$ be an integer and suppose $a_{L}$ and $b_{L}$ in L satisfy $-1 / 4+n<a_{L}<b_{L}<n$ or $1 / 4+n<a_{L}<b_{L}<1 / 2+n$. There is an expression of the form $Y X$ mapping $a_{L}$ to $n$ and $b_{L}$ to $1 / 4+n$.

Proof. Without loss of generality, suppose $-1 / 4+n<a_{L}<b_{L}$ $<n$. For each $x \in(-1 / 4+n, n)$, there is a unique $t_{1}$ such that $\left(\exp t_{1} X\right)\left(a_{L}\right)=x$; let $\left(\exp t_{1} X\right)\left(b_{L}\right)=b_{L}(x)$ and notice that $-1 / 4+n$ $<x<b_{L}(x)<n$. There is a unique $t_{2}$ such that $\left(\exp t_{2} Y\right)(x)=n$; let $\left(\exp t_{2} Y\right)\left(b_{L}(x)\right)=b_{L}(x)$ and notice that $n<b_{L}(x)<n+1 / 2$. We shall prove that when $x \rightarrow n, b_{L}(x) \rightarrow n$ and when $x \rightarrow n-1 / 4, b_{L}(x) \rightarrow n$ $+1 / 2$; by continuity there is an $x$ with $b_{L}(x)=n+1 / 4$.

We have 


$$
\begin{aligned}
& \left\langle\tau(x), \tau\left(b_{L}(x)\right) ; \alpha-\left(\alpha^{2}-1\right)^{1 / 2}, \alpha+\left(\alpha^{2}-1\right)^{1 / 2}\right\rangle \\
& \quad=\left\langle\infty, \tau\left(b_{L}(x)\right) ; \alpha-\left(\alpha^{2}-1\right)^{1 / 2}, \alpha+\left(\alpha^{2}-1\right)^{1 / 2}\right\rangle
\end{aligned}
$$

so

$$
\begin{gathered}
\frac{\tau(x)-\left(\alpha-\left(\alpha^{2}-1\right)^{1 / 2}\right)}{\tau(x)-\left(\alpha+\left(\alpha^{2}-1\right)^{1 / 2}\right)} \cdot \frac{\tau\left(b_{L}(x)\right)-\left(\alpha+\left(\alpha^{2}-1\right)^{1 / 2}\right)}{\tau\left(b_{L}(X)\right)-\left(\alpha-\left(\alpha^{2}-1\right)^{1 / 2}\right)} \\
=\frac{\tau\left(b_{L}(x)\right)-\left(\alpha+\left(\alpha^{2}-1\right)^{1 / 2}\right)}{\tau\left(b_{L}(x)\right)-\left(\alpha-\left(\alpha^{2}-1\right)^{1 / 2}\right)} .
\end{gathered}
$$

As $x \rightarrow n, \tau(x) \rightarrow \infty$ and the first factor on the left approaches 1 . Since $x<b_{L}(x)<n$, as $x \rightarrow n, b_{L}(x) \rightarrow n$ and $\tau\left(b_{L}(x)\right) \rightarrow \infty$, so the second factor on the left approaches 1 . It follows that as $x \rightarrow n, \tau\left(b_{L}(x)\right) \rightarrow \infty$ and $b_{L}(x) \rightarrow n$.

Similarly

$$
\begin{aligned}
& \left\langle\tau(x), \alpha-\left(\alpha^{2}-1\right)^{1 / 2} ; \tau\left(b_{L}(x)\right), \alpha+\left(\alpha^{2}-1\right)^{1 / 2}\right\rangle \\
& \quad=\left\langle\infty, \alpha-\left(\alpha^{2}-1\right)^{1 / 2} ; \tau\left(b_{L}(x)\right), \alpha+\left(\alpha^{2}-1\right)^{1 / 2}\right\rangle
\end{aligned}
$$

so

$$
\begin{gathered}
\frac{\tau(x)-\tau\left(b_{L}(x)\right)}{\tau(x)-\left(\alpha+\left(\alpha^{2}-1\right)^{1 / 2}\right)} \cdot \frac{-2\left(\alpha^{2}-1\right)^{1 / 2}}{\left(\alpha-\left(\alpha^{2}-1\right)^{1 / 2}\right)-\tau\left(b_{L}(x)\right)} \\
=\frac{-2\left(\alpha^{2}-1\right)^{1 / 2}}{\left(\alpha-\left(\alpha^{2}-1\right)^{1 / 2}\right)-\tau\left(b_{L}(x)\right)} .
\end{gathered}
$$

As $x \rightarrow n, \tau(x) \rightarrow \infty$ and the first factor on the left approaches 1 . Since map, $\tau\left(b_{L}(x)\right) / \tau(x)=\tau\left(b_{L}\right) / \tau\left(a_{L}\right)$. Therefore as $x \rightarrow-1 / 4+n, \tau\left(b_{L}(x)\right)$ $\rightarrow \tau\left(b_{L}\right)\left(\alpha+\left(\alpha^{2}-1\right)^{1 / 2}\right) / \tau\left(a_{L}\right)$ and the cross-ratio approaches $\infty$; it follows that $\tau\left(b_{L}(x)\right) \rightarrow \alpha-\left(\alpha^{2}-1\right)^{1 / 2}$ and hence $b_{L}(x) \rightarrow n+1 / 2$.

LEMMA 18. If $k \geqq 3$ is odd, the order of generation of $\widetilde{S L}(2, R) / k Z$ with respect to $X, Y$ is $k+2$.

Proof. Let $(a, b, c, d)$ be a 4-tuple as described above. Choose $a_{L}$ and $b_{L}$ in $L$ covering $a$ and $b$ and suppose for a moment that $-1 / 4$ $<a_{L}<b_{L}<1 / 2$. Let $n$ be an integer satisfying $|n| \leqq(k-1) / 2$. The reader can easily show that an expression with $k-1$ terms of the form $X Y \cdots X Y$ exists mapping $a_{L}$ and $b_{L}$ to $\tilde{a}_{L}$ and $b_{L}$ where $-1 / 4$ $+n<\tilde{a}_{L}<\tilde{b}_{L}<n$ or $1 / 4+n<\tilde{a}_{L}<b_{L}<1 / 2+n$. By lemma 17, there is an expression of the form $\widetilde{Y X}$ mapping $\tilde{a}_{L}, b_{L}$ to $n, 1 / 4+n$; then $(\widetilde{Y X})(X Y \cdots X Y)=\tilde{Y}(\tilde{X} X)(Y \cdots X Y)$ is an expression of length $k$ mapping $a_{L}$ to $n$ and $(a, b)$ to $(\infty, 0)$. Since the image of $c$ and $\alpha-$ $\left(\alpha^{2}-1\right)^{1 / 2}$ belong to the same component of $P^{1}-\{0, \infty\}$ (because all triples are oriented), we can find an element of $\exp (t X)$ leaving $\infty$ and 
0 fixed and mapping the image of $c$ to $\alpha-\left(\alpha^{2}-1\right)^{1 / 2}$; thus we can find an expression of length $k+1$ mapping $(a, b, c)$ to $(\infty, 0, \alpha-$ $\left.\left(\alpha^{2}-1\right)^{1 / 2}\right)$ and $a_{L}$ to $n$. By previous remarks, this proves the lemma; since only $k+1$ terms have been used, we have an extra term at our disposal with which to force the original assumption on $a_{L}$ and $b_{L}$.

Finally, suppose $a$ and $b$ are arbitrary. Choose $a_{L}$ and $b_{L}$ in $[0,1)$ covering $a$ and $b$. If $a_{L}<b_{L}$, an expression of the form $\exp (t X)$ exists mapping both into the interval $[0,1 / 2)$ and the above argument takes over from there. If $0 \leqq b_{L} \leqq 1 / 4<a_{L}<1$, an expression of the form $\exp (t X)$ exists leaving $b_{L}$ in $[0,1 / 4]$ and mapping $a_{L}$ into $(3 / 4,1)$; this last point is equivalent to a point in $(-1 / 4,0)$, so the previous argument takes over once more. We are done unless $0 \leqq b_{L}<a_{L} \leqq 1 / 4$ or $1 / 4<b_{L}<a_{L}<1$.

Similar arguments hold for the pair $(c, d)$. In this case we choose $c_{L}, d_{L}$ in $(-1 / 4,3 / 4]$; we are done unless $-1 / 4<d_{L}<c_{L}<1 / 2$ or $1 / 2 \leqq d_{L}<c_{L} \leqq 3 / 4$. But $(a, b, c, d)$ is an oriented 4-tuple, so $0 \leqq b_{L}$ $<a_{L} \leqq 1 / 4$ implies $0 \leqq b_{L}<c_{L}<d_{L}<a_{L} \leqq 1 / 4$ and we are done; $1 / 2 \leqq d_{L}<c_{L} \leqq 3 / 4$ implies $1 / 2 \leqq d_{L}<a_{L}<b_{L}<c_{L} \leqq 3 / 4$ and we are again done. We can have trouble only if $1 / 4<b_{L}<a_{L}<1$ and $-1 / 4<d_{L}<c_{L}<1 / 2$. In this case if $c_{L}<0, d_{L}+1$ and $c_{L}+1$ are the unique representatives of $c$ and $d$ in $[0,1)$; then $b_{L}<c_{L}+1<$ $d_{L}+1<a_{L}$, contradicting $d_{L}<c_{L}$. If $d_{L} \geqq 0, c_{L}$ and $d_{L}$ are the representatives of $c$ and $d$ in $[0,1)$ and again $c_{L}<d_{L}$. Hence $-1 / 4<d_{L}<0$ and $0 \leqq c_{L}<1 / 2$; then $c_{L}$ and $d_{L}+1$ are the unique representatives of $c$ and $d$ in $[0,1)$, so $b_{L}<c_{L}<d_{L}+1<a_{L}$, and $b_{L} \in(1 / 4,1 / 2), a_{L} \in(3 / 4,1)$. Therefore $a$ has a second representative $\tilde{a}_{L}$ in $(-1 / 4,0)$ and the arguments given earlier apply to $(a, b)$.

LEMMA 19. If $k \geqq 2$ is even, the order of generation of $\widetilde{S L}(2, R) / k Z$ with respect to $X, Y$ is $k+2$.

Proof. Let $(a, b, c, d)$ be a 4-tuple of the usual kind, and choose $a_{L}$ and $b_{L}$ covering $a$ and $b$. Suppose for a moment that $1 / 4<a_{L}<b_{L}$ $<1$. Let $n$ be an integer satisfying $-k / 2<n \leqq k / 2$. The reader can easily show that an expression of the form $X Y \cdots Y X$ with length $k-1$ exists mapping $a_{L}$ and $b_{L}$ to $\tilde{a}_{L}$ and $\tilde{b}_{L}$ where $-1 / 4+n<\tilde{a}_{L}<b_{L}$ $<n$ or $1 / 4+n<\tilde{a}_{L}<b_{L}<1 / 2+n$. By lemma 17 , there is an expression of the form $Y X$ mapping $\tilde{a}_{L}$ and $b_{L}$ to $n$ and $1 / 4+n$; $(\widetilde{Y X})(X Y \ldots$ $Y X)=\tilde{Y}(\tilde{X} X)(Y \cdots X)$ is an expression of length $k$ mapping $a_{L}$ and $b_{L}$ to $n$ and $1 / 4+n$. From here on, the proof follows that given for lemma 18.

Suppose next that $a$ and $b$ are arbitrary; choose $a_{L}$ and $b_{L}$ in $(-1 / 4$, 3/4] covering $a$ and $b$. If $a_{L}<b_{L}$, an expression of the form $\exp (t Y)$ maps both into the interval $(1 / 4,3 / 4]$ and the previous argument takes 
over. If $-1 / 4<b_{L}<1 / 2 \leqq a_{L} \leqq 3 / 4$ an expression of the form $\exp (t Y)$ leaves $a_{L}$ in $[1 / 2,3 / 4]$ and maps $b_{L}$ into $(-1 / 4,0)$; this last point is equivalent to a point in $(3 / 4,1)$ and the previous argument takes over again. We are done unless $-1 / 4<b_{L}<a_{L}<1 / 2$ or $1 / 2 \leqq b_{L}<a_{L}$ $\leqq 3 / 4$.

Similar arguments hold for the pair $(c, d)$. In this case we choose $c_{L}, d_{L} \in[0,1)$ and we are done unless $0 \leqq d_{L}<c_{L} \leqq 1 / 4$ or $1 / 4<d_{L}$ $<c_{L}<1$. By an argument similar to that of lemma 18 , both bad situations can occur only if $a_{L} \in(1 / 4,1 / 2)$ and $b_{L} \in(-1 / 4,0)$. But then $b$ has a second representative $b_{L} \in(3 / 4,1)$ and earlier arguments apply to $(a, b)$.

Remarx. Finally, consider the case

$$
X=\left(\begin{array}{rr}
1 & 0 \\
0 & -1
\end{array}\right), \quad Y=\left(\begin{array}{rr}
1 & 2 \\
0 & -1
\end{array}\right), \quad Z=\left(\begin{array}{rr}
1 & 0 \\
-2 & -1
\end{array}\right) .
$$

The fixed points of $\exp (t X)$ are $0, \infty$, those of $\exp (t Y)$ are $-1, \infty$, and those of $\exp (t Z)$ are $-1,0$. We suppose $\tau(1 / 3)=-1, \tau(2 / 3)=0$.

Any two of $X, Y, Z$ generate a two-dimensional subgroup; the order of generation of all two-dimensional Lie groups is two [5]. Consequently, if an expression equals $g$ in $\widetilde{S L}(2, R)$ and contains three consecutive terms from the same pair, there is a shorter expression which also equals $g$. A little thought shows that we can restrict attention to expressions in which $X, Y$, and $Z$ appear cyclically.

LEMMA 20. The order of generation of $\widetilde{S L}(2, R)$ with respect to $X, Y, Z$ is $\infty$. If $k \geqq 2$ is even, the order of generation of $\widetilde{\mathrm{SL}}(2, R) / k Z$ with respect to $X, Y, Z$ is at least $(3 k+6) / 2$. Moreover, no expression of length $(3 k+6) / 2$ can give all of $\widetilde{S L}(2, R) / k Z$.

Lemma 20. The order of generation of $S L(2, R)$ with respect to

Proof. Consider the expression $Y X \cdots Z Y X$ of length $(3 k+6) / 2-1$ and suppose it gives $e \in P S L(2, R)$. In $L, X(0)=0, Y X(0)=0$, $\operatorname{ZYX}(0)<1 / 3, \operatorname{XZYX}(0)<2 / 3$, etc., so the image of 0 is smaller than $k / 2$. Similarly $\operatorname{ZYX}(0)>-1 / 3, \operatorname{XZYX}(0)>-1 / 3, \operatorname{YXZYX}(0)>-2 / 3$, $\operatorname{ZYXZYX}(0)>-2 / 3$, etc., so the image of 0 is considerably larger that $-k / 2$. Hence the expression acts on $L$ by $x \rightarrow x+n$ where $|n|<k / 2$. The same result holds for any cyclic expression of length $(3 k+6) / 2$ -1 . It follows that no combination of expressions of length less than $(3 k+6) / 2$ can give $k$ points in $\pi^{-1}(e)$.

Even the expression $Z Y X \cdots Z Y X$ of length $(3 k+6) / 2$ does not give every element of $\widetilde{S L}(2, R) / k Z$. Indeed the image of 0 in $L$ under this expression belongs to the interval $(-k / 2+1 / 3, k / 2+1 / 3)$; this interval contains only $k-1$ points equivalent to $1 / 3$. Thus if $g \in P S L(2, R)$ maps $(\infty,-1,0)$ to $(-1,0, \infty)$, the expression $\cdots$ YXZYX of length $(3 k+6) / 2$ gives at most $k-1$ points in $\pi^{-1}(g)$. 
LEMMA 21 . If $k \geqq 3$ is odd, the order of generation of $\widetilde{S L}(2, R) / k Z$ with respect to $X, Y, Z$ is at least $(3 k+5) / 2$. Moreover, no expression of length $(3 k+5) / 2$ can give all of $\widetilde{\mathrm{SL}}(2, R) / k Z$.

Proof. Let $g$ in $\operatorname{PS} L(2, R)$ map $(\infty,-1,0)$ to $(0, \infty,-1)$. Some thought shows that we can choose $\tau$ so the map from $L$ to $L$ given by $x \rightarrow x+2 / 3$ covers $g$. Consider the expression $Z Y X \cdots Z Y X$ of length $(3 k+5) / 2-1$ and suppose it gives $g$ in $\operatorname{PS} L(2, R)$. In $L$, the image of 0 is contained in $(-(k-1) / 2-1 / 3,(k-1) / 2+1 / 3)$. Hence the action of the expression on $L$ is $x \rightarrow x+\lambda$ where $|\lambda|<(k-1) / 2+$ 1/3. A similar result holds for any cyclic expression of length $(3 k+5) / 2$ -1 . But there are only $k-1$ numbers in $(-(k-1) / 2-1 / 3,(k-1) / 2$ $+1 / 3$ ) equivalent to $2 / 3$.

Even the expression $X Z Y X \cdots Z Y X$ of length $(3 k+5) / 2$ does not give $k$ points in $\pi^{-1}(g)$, for in $L$ the image of 0 is, in fact, less than $(k-1) / 2$ $+2 / 3$ and larger than $-(k-1) / 2$, and this interval contains only $k-1$ points equivalent to $2 / 3$.

Lemma 22. Let $(a, b, c)$ be an oriented triple, and let $a_{L}$ in $L$ cover a. Suppose there is an expression of length $\ell$ taking $A_{L}=0$ to $a_{\ell}$. Then there is an expression of length $\ell+2$ taking $A_{L}$ to $a_{L}$ and $(\infty,-1,0)$ to $(a, b, c)$.

Proof. Let the inverse of the expression in question map $(a, b, c)$ to $(\infty, \tilde{c}, \tilde{c})$; it is enough to find an expression with two terms fixing $A_{L}$ and mapping $(\infty,-1,0)$ to $(\infty, \tilde{b}, \tilde{c})$.

If $-1<c$, there is an element in $\exp (t Y)$ mapping 0 to $\tilde{c}$. If this expression maps $\tilde{\tilde{b}}$ to $\tilde{b}$, it maps $(\infty, \tilde{b}, 0)$ to $(\infty, \tilde{b}, \tilde{c})$; since all triples are oriented, $\tilde{\tilde{b}}<0$ and there is an element in $\exp (t X)$ mapping -1 to $\tilde{\tilde{b}}$, so $Y X$ maps $(\infty,-1,0)$ to $(\infty, \tilde{b}, \tilde{c})$.

If $\tilde{c} \leqq-1, b<\tilde{c}<0$ and there is an element in $\exp (t X)$ mapping -1 to $\tilde{b}$. Let this expression map $\tilde{\tilde{c}}$ to $\tilde{c}$; then $(\infty,-1, \tilde{\tilde{c}})$ maps to $(\infty, \tilde{b}, \tilde{c})$, so $-1<\tilde{\tilde{c}}$. Hence there is an element in $\exp (t Y)$ mapping 0 to $\tilde{\tilde{c}}$ and $X Y$ maps $(\infty,-1,0)$ to $(\infty, \tilde{b}, \tilde{c})$.

LEMMA 23. Let $k \geqq 2$ be even and let $\left|a_{L}\right| \leqq k / 2$; there is an expression of length $3 k / 2+1$ mapping $A_{L}=0$ to $a_{L}$. Hence the order of generation of $\widetilde{S L}(2, R) / k Z$ is $(3 k+6) / 2$.

Proof. If $a_{L}>0$, the expression $Z Y X \cdots Y X Z$ suffices; indeed $Z(0)$ can be any point in $[0,1 / 3), X Z(0)$ can be any point in $[0,2 / 3)$, etc. If $a_{L}<0$, the expression $Z X Y \cdots X Y Z$ similarly suffices.

LEMMA 24. Let $k \geqq 3$ be odd and let $\left|a_{L}\right| \leqq k / 2$; there is an expression of length $(3 k+1) / 2$ mapping $A_{L}=0$ to $a_{L}$. Hence the order of generation of $\widetilde{S L}(2, R) / k Z$ is $(3 k+5) / 2$. 
Proof. Exactly as for lemma 23.

\begin{tabular}{|c|c|c|c|c|c|c|c|}
\hline & & & & $\begin{array}{c}\text { Order of } \\
\text { Generation } \\
k=0\end{array}$ & $\begin{array}{c}\text { Order of } \\
\text { Generation } \\
k=1\end{array}$ & $\begin{array}{c}\text { Order of } \\
\text { Generation } \\
k \geqq 2\end{array}$ & $\begin{array}{l}\text { Expressions } \\
\text { Giving All } \\
\quad \text { of } G\end{array}$ \\
\hline elliptic: & $\left(\begin{array}{rr}0 & -1 \\
1 & 0\end{array}\right)$ elliptic: & $\left(\begin{array}{cc}0 & \alpha \\
1 & 0\end{array}\right)$ & - & $\infty$ & $\infty$ & $\infty$ & - \\
\hline elliptic: & $\left(\begin{array}{rr}0 & -1 \\
1 & 0\end{array}\right)$ parabolic: & $\left(\begin{array}{ll}0 & 1 \\
0 & 0\end{array}\right)$ & - & 3 & 3 & 3 & $X Y X$ \\
\hline elliptic: & 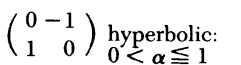 & $\left(\begin{array}{ll}0 & \alpha \\
1 & 0\end{array}\right)$ & - & 3 & 3 & 3 & $X Y X$ \\
\hline parabolic: & $\left(\begin{array}{ll}0 & 1 \\
0 & 0\end{array}\right)$ parabolic: & $\left.\begin{array}{l}0 \\
0\end{array}\right)$ & - & $\infty$ & 4 & $k+2$ & $\begin{array}{l}\cdots X Y X \text { and } \\
\cdots Y X Y\end{array}$ \\
\hline parabolic: & $\left(\begin{array}{ll}0 & 1 \\
0 & 0\end{array}\right)$ hyperbolic: & $\left(\begin{array}{ll}0 & 1 \\
1 & 0\end{array}\right)$ & - & $\infty$ & 4 & $k+2$ & $\begin{array}{l}\cdots X Y X \text { and } \cdots Y X Y \\
\text { if } k=1 \text { or } k \text { even; } \\
X \cdots X \text { if } k \geqq 3 \\
\text { and } k \text { odd }\end{array}$ \\
\hline hyperbolic: & $\begin{aligned}\left(\begin{array}{rr}1 & 0 \\
0 & -1\end{array}\right) & \text { hyperbolic: } \\
& \text { (fixed points } \\
& \text { non-interlac- } \\
& \text { ing) } \alpha>1:\end{aligned}$ & $\left(\begin{array}{ll}\alpha & -1 \\
1 & -\alpha\end{array}\right)$ & - & $\infty$ & 4 & $k+2$ & None \\
\hline hyperbolic: & 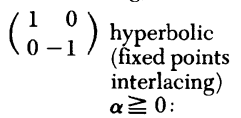 & $\left(\begin{array}{rr}\alpha & 1 \\
1 & -\alpha\end{array}\right)$ & - & $\infty$ & 6 & $2 k+4$ & $\begin{array}{l}\cdots X Y X \text { and } \\
\cdots Y X Y\end{array}$ \\
\hline & $\left(\begin{array}{rr}1 & 0 \\
0 & -1\end{array}\right)$ & $\left(\begin{array}{rr}1 & 2 \\
0 & -1\end{array}\right)$ & $\left.\begin{array}{rr}1 & 0 \\
-2 & -1\end{array}\right)$ & $\infty$ & 4 & $\begin{array}{l}\frac{3 k+5}{2} \text { for } \\
k \text { odd; } \\
\frac{3 k+6}{2} \text { for } \\
k \text { even }\end{array}$ & None \\
\hline
\end{tabular}

\section{REFERENCES}

1. G. Hochschild, The Structure of Lie Groups, Holden-Day, San Francisco, 1965.

2. R. M. Koch and F. Lowenthal, Uniform Finite Generation of Three Dimensional Linear Lie Groups, Can. J. Math. 27 (1975), 396-417.

3. F. Lowenthal, Uniform finite generation of the isometry groups of Euclidean and non-Euclidean geometry, Can. J. Math. 23 (1971), 364-373.

4. - Uniform finite generation of the rotation group, Rocky Mountain J. Math. 1 (1971), 575-586.

5. —, Uniform finite generation of the affine group, Pacific J. Math. 40 (1972), 341-348.

6. — Uniform finite generation of $S U(2)$ and $S L(2, R)$, Can. J. Math. 24 (1972), 713-727.

7. H. Yamabe, On an arcwise connected subgroup of a Lie group, Osaka J. Math. 2 (1950), 13-14.

University of Oregon, Eugene, Oregon 97403

University of Wisconsin at Parkside, Kenosha, Wisconsin 53140 